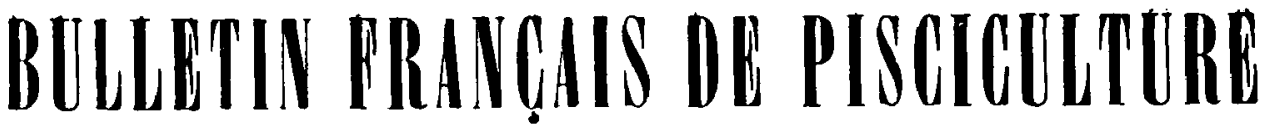

\section{LA MOULE MIGRATRICE \\ (Dreissensia polymorpha Pall.) \\ DANS LE LAC BALATON}

\author{
Par le Docteur Charles LUKACS \\ Directeur de Ia Balaton Llalaszati Részvénytarsasag.
}

La Mreissensia cu Moule migratrice est originaire de l’Grient, comme lo Lézard (Lacerta praticola). La Tortue (Testudo graeca) ou les poissons devenus si mportants dans le Balitun, la Carpe el le Sandre de pierre (Lucioperce volgensis). La rapidité de son expansion, sa pullulation extraordinaire reppelle colle du hat migrateur, de provenance également orientale.

En Europe, les premiers exemplaires de la Moule migratrice ont été trouvés, en I'an 1768 , dans les fleures de la Russie méridionale. La première description scientifique de l'espèce ful donnée par Pallas en 177 I. Son apparition dans l'Europe centrale a été signalée par BaEn, qui, en I 824, trouvait des masses de Dreissensia dans les Hafl's allemands. En I826, on la rencontre dans la Havel, près Polsdam et, ell mème temps, dans le Bas-Phin. à Huningue. Attachée sur le corps des navires, on la découvie bientôt dans le Haut-Rhin, à Mannheim.

Ce fut aussi par l’intermédiaire de bateaux, spécialement de navires hollandais, qu'elle semble avoir ćlé importée en France et en Ingleterre (1). Sclon Latiterisorx, elle passe par le canal Danube-Mein dans le Danube, où on la rencontre pour la première fois à Ratisbonne, en s868. Dans le Danube hongrois, elle devait arriser du sens opposé, par le Bas-Danube, car Guossixan l'avait trouvé, dès l’an r79o, dans quelques rivières honcrroises, appartenant au bassin du Danube (Zsika, liudoesér).

Juscfu'il y a trois ans, personne ne connaissait encore cette espèce de moule dans notre " mer hongroise ". Elle était - depuis I856 (Frivaldszkx) - bien répandue dans le Danube mìme, oì clle fut imporlée, à coup) sûr, par des navires, de la région dilc "Ponlique " (Ponlus Euxinus), mais elle ne figrurait pas du tout dans la faune vivante de notre grand lac.

C'est en Octobre s.3\% que les savants de l'Instilut de Biologie de Tihany

(I) Kurt LAmpert : - Das Leben der Binnengewaesser. III Ld., 1925, p. 93. 
(M. le Professeur Geza Ery, directeur de l'Institut et son assistants Mlle Olga Srbestráa) ont reconnu ce petit invertébré adhérent à une moule d'étang qui venait de blesser le fied d'un baigneur, hydrobiologiste luimême ( $\mathrm{D}^{\mathrm{r}}$ llur) dans la baie de Tihany. En 1933 , du printemps à l'automne, on trouva ce parasite sur plus de $4 \%$ des Inionidés ramassés. Le Directeur de l'Institut me fit part de l'apparition de ce Mollusque, en me priant d'attirer l'attention de nos pècheurs sur ce nouveau " colon " de notre lac et de leur ordonner d'en rassembler, pendant les pèches, de tous les coins du Balaton. Je me mis au I ravitil moi-mème pour en recueillir des spécimens intéressants daus la zome liltorale de la rive méridionale, et je pus constater que - aw (nvirons de Siofok - s sur so des moules Uni')

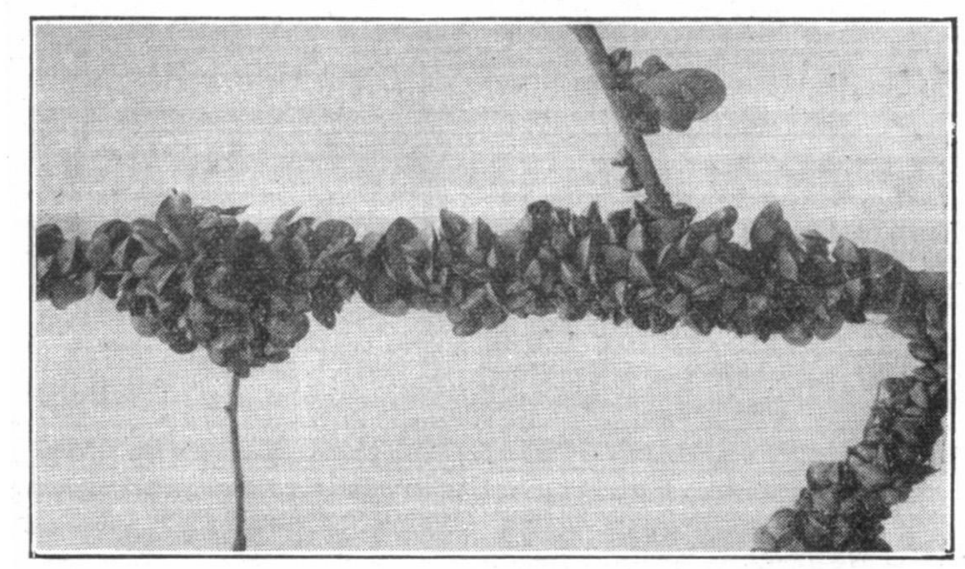

Figi. 7. - Branclie de saule couverte de Dreissensia.

pictorum et Anodonta cygnea portaient, sulidement atlachés avec leurs touffes de byssus, de à r 2 individus de Dreissensia.

Pendant l'été de 1934, nos maîtres-pècheurs apportèrent, l'un après l'autre, des Ironcs de bois, branches d'arbres immergées, touffes de Potamots. bloes de pierres, relevés de la vase du lac, tous portant une quantite considérable de Dreissensia (Fig. 7). - La propagation de ce nouveau Mollusque commençaient à inquiéter les gens naviguant sur le lac, car tous les bateaux tirés de l'eau ćtaient, au fond, converts de milliers de ce bivalve Iriangulaire. D'abord, ce ful seulement dans le bassin occidental, entre Kenese et Tihany, surlout aux cnvirons de l'embouchure du canal de Sió, que les Mollusques migrateurs se montraient en nombre toujours croissant, mais vers l'automne 1934 nous en trouvâmes peu à peu aussi dans le bassin occidental, et enfin la Dreissensia apparut même dans la baie de Keszthely, le coin le plus éloigné de Siólok. Dans les deux premières années après leur découverte, la Dreissensia ne se trouvait pas sur d'autres membres de la faune balatonienne que ses similaires, les Unionidés, à partir de l'été 1934, nos pècheurs pèchèrent aussi quelques Ecrevisses (Astacus 


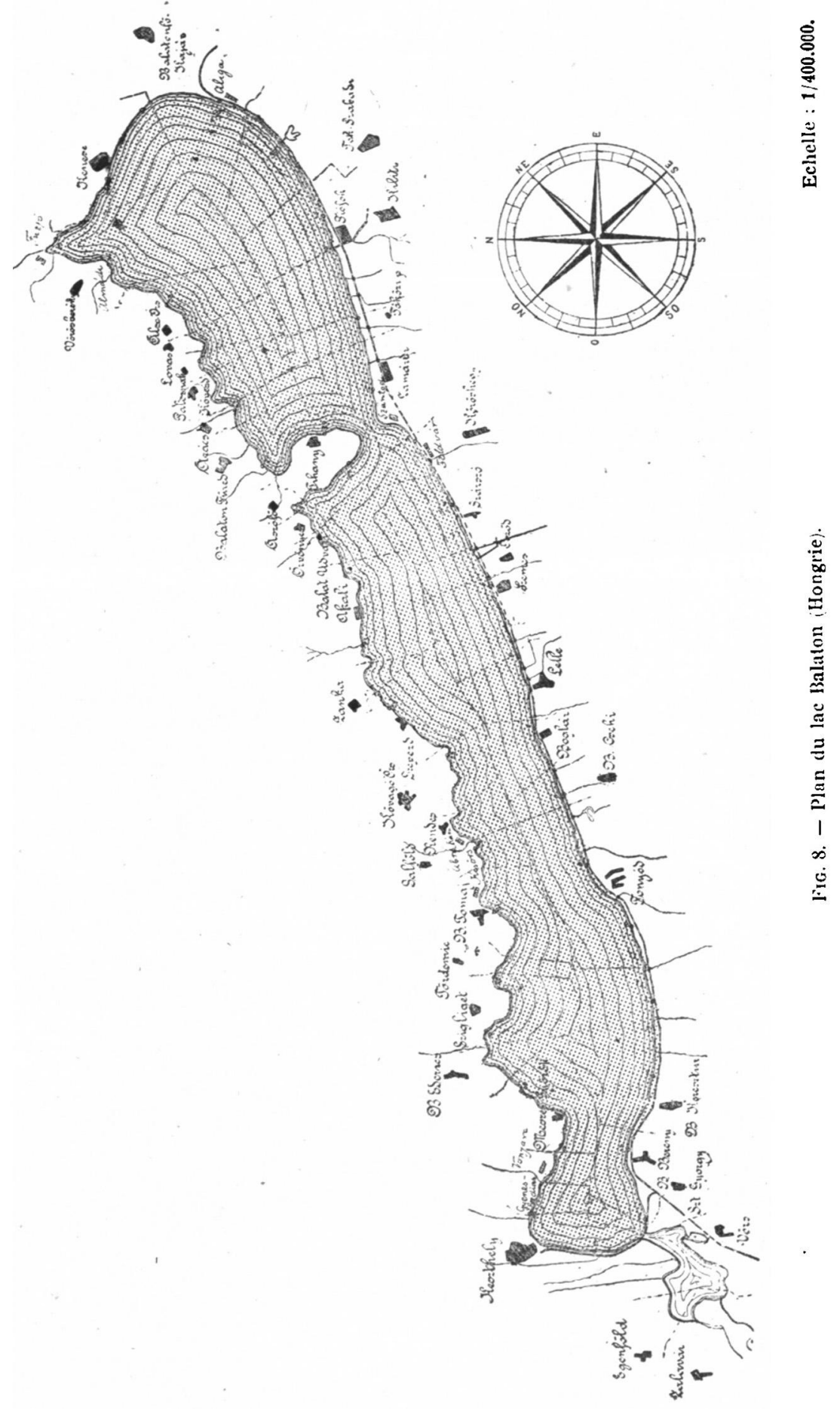


leptodactylus) bien développées, qui étaient envahies par ce parasite (Fig. 9). Je me suis empressé d'envoyer quelques spécimens de ces petits monstres à M. le Professeur Extz à Tihany, qui - à l'aide de son assistante $M^{\text {lle }}$ Srbestréx - était en train de préparer un travail scientifique bien documenté sur l'expansion de ce nouveau membre de la faune du lac. Au cours de l'observation des Eerevisses pêchées depuis la capture du premier individu hôte de Dreissensia, je pus faire la constatation que, pendant la saison d'été ig3/, à peu près un demi pour cent des Ecrevisses prises par nos filets furent trouvées infectées par ce parasile.

La grandeur de ce Mollusque varie dans notre lac entre 3 et 30 millimètres, la plupart ne dépassent pas 10 millimètress. Parmi les milliers ef milliers d’individus qui passèrent intre les mains de $\mathbf{M}^{\mathrm{uc}}$ Sebestróx, jusquea Octobre dernier, un seul alteignit 35 millimètres. Le Dreissensin se multi. plie par des larves (trophophores) crrant librement dans l'eau.

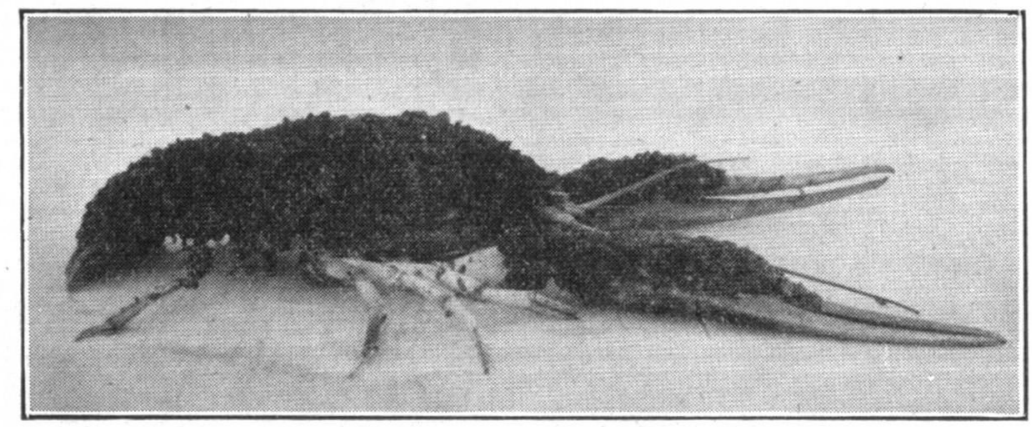

Fig. 9. - Eerevisse à patt:'s grèles couverte de Dressensia.

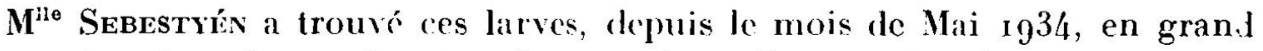
nombre dans le zooplancton de notre lac, elles sont incolores et transparentes, avec des coquilles reconnaissables; leur longueur est d'environ roo microns $(\mu)$. Vers la fin de Juin, le jenne Mollusque acquiert sa forme triangulaire, descend vers le fond el sattache avec son byssus sur des pierres, des tiges de Phragmites ot de Potamots, des coquilles d'Unionidés, etc.

L'introduction de ce nouvel invertébré dans notre lac est due, sans nul doute, à une "migration passive", par l'intermédiaire de bateaux venus, à travers le canal de Sió, du fleuse Danube. ('est vers rg3o qu'un grand bateau à remorque, bâti en fer, fut monté, à l'ícluse du Sió, dans le Balaton. Le transport ne dura que 3 à 4 jours. Persomne ne pouvait imaginer alors que ce véhicule aquatique serait l'agent intermédiaire de l’immigration du Dreissensia dans le lac Balaton. Quand, en Juillet dernier, on fit sortir de nouveau ce batcau, long de aj mètres, sa parlic inféricure et tout son fond étaient littéralement couverts d'une masse énorme de Dreissensia. Outre ce grand bateau à remorque, un grand nombre de canots spor- 
tifs furent montés du Danube dans le Balaton, el ce fait explique clairement par quel moyen le Hollusque migralcur put envahir notre grand lae.

Pour nous, praticiens de priche, la grande question n'est pas celle de l'origine, mais celle du rôle de ce Mollusque dans l'économie piscicole. II est bien possible que les Lnionidés auxquels vivent attachées pendant des mois des dizaines de ces parasites, deviennent à la longue les victimes de ces intrus, et le minc solt altend probiblement aussi les malhcureuses Érevisses auxquelles s'attaque leur essaim collant.

Jusqu'à ce jour, nous n'avons rien aperçu qui justifierait la crainte que la rapide propagation de ce Mollusque oriental portât à la fin préjudice sérieux à nos poissons ou bien aux organismes leur servant d'aliment. Cette question ne pourra être élucidée qu à l’aide de recherches laborieuses sur les contenus stomacaux. Dans son dernier rapport sur la Dreissensia, M $^{\text {He }}$ Srebestrúx signnale déjà avoir trousé quelques jeunes exemplaires de lia moule migratrice dans l'estomar de guclques perches et dans les intestins (i un certain nombre d'Ablettes (1lburnus lucidus).

Cette étude est poursuivie d'une manière méthodique et consciencieuse par les érudits de l'Institut de Biologie de Tihany, et nous avons toute raison à attendre de ce côté des renseignements propres à enrichir nos connaissances et - en mimo tomps - a nous servir de liğne de conduite dans l'intérêt de l'économie piscicole de notre lac (I).

\section{BIPLIOGRAPHIE:}

Kurt Laypent : - Das Leben der Binnengewaesser, r 9:5. III éd.

G. ENTz - Arbeiten des Biol. Forschungsinstiluts von Tihany. Sonderabdruck des Mathem. und Naturwiss. Anzeiger der Lngar. Akad. der Wiss. - Budapest, ig34.

O. Sebsstén: - Appearance and rapirl increase of Dreissensia polymorpha... in lake Balaton. - Eturle parue dans le Vol. VI des Travaux de l'Institut de Biol. de Tihany, 1934. - Texte hongrois avec extrait anglais.

Ch. Lthacs : - Vindorkagyló a Balatonban. - Moule migratrice dans le lac Balaton. - Haliszat, Mai $193 \hat{\imath}$.

l: Uxgan: -- Mit Wandermuscheln besctzte hrebse im Balatonsce. - Halászat, Décembre ig34.

Le mème article en allemand dans Ofsterreichs Fischereiwirlschuf, Janvier 1935.

(1) Nous croyons devoir signaler que dans les derniers jours de Juillet, nous avons ike informés far un pêcrheur sporpif, digne de foi, qu'à tmis reprises. sa femme a trouvó dans les intestins des Carpes par lui capturées, quantitó de Moules migratrices bien reconnaissables. Il est à remarquer que, malgré les amorçages au maïs, la pêche

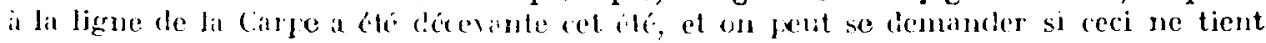
pas a ce que ce poisson trouve dans la Dreissensia une pâture abondante et à son goùt. 